\title{
Early Antenatal Sonographic Findings of Rubinstein-Taybi Syndrome: Imaging of High-Arched Palate and Bilateral Abducted Thumbs on Surface Rendering Mode at 17 Weeks
}

\section{Introduction}

Rubinstein-Taybi Syndrome (RSTS) is a rare genetic disorder (estimated birth prevalence 1 in 100,000 to 125,000). Rubinstein and Taybi originally described seven children with typical dysmorphic facial features, broad thumbs and toes, and varying degrees of mental retardation. The syndrome is associated with multiple systemic anomalies. The literature includes about a thousand reported cases since 1963 . This condition is inherited in an autosomal dominant pattern, but the majority of cases are derived by de novo mutation. It may be caused by micro deletion within $16 \mathrm{p} 13.3$ or different mutations in the genes encoding the transcriptional coactivator CREB-binding protein and E1A-binding protein p300. The cytogenetic anomalies of RSTS remain unknown in about $37 \%$ of patients. The diagnosis is based on clinical presentation during the early postnatal period (Spena $S$ et al. J Pediatr Genet 2015; 4: 177-186).

Only three cases of antenatal ultrasound diagnosis or detection have been reported to date. Here we present the ultrasound features of a new case.

\section{Case Report}

A 30-year-old secundigravida with a family history of consanguinity declined chorion villus sampling and karyotyping for increased nuchal translucency $(3.4 \mathrm{~mm}$ at crown rump length of $48.1 \mathrm{~mm}$ ) and high risk at combined maternal serum testing in the first trimester $(1 / 10)$.

We performed serial examinations by 2D and 3D ultrasound, using GE Voluson ${ }^{\circledR}$ E8 (GE Medical Systems, Zipf, Austria)ultrasound machine equipped with a RAB6-D transducer. The 3D surface rendering mode at 17 weeks suggested the presence of hypotelorism and a high-arched palate. The reconstruction of the palate, using a $3 \mathrm{D}$ surface rendering mode at 17 weeks, fails to demonstrate its classic delta shape ( Fig. 1). The small oral cavity and acoustic shadowing blocked out the correct visualization of the palate at 22 and 32 weeks. Retrospective analysis of the sonographic images revealed bilateral abducted and thick thumbs at 12 and 17 weeks ( $>$ Fig. 2). We found partial agenesis of the corpus callosum at the 22- and 32-week scan. Decline in fetal growth and cryptorchidism occurred at the 32-week scan.

No other structural abnormalities were observed. The patient refused to be referred for fetal brain magnetic resonance imaging (MRI), genetic counseling and amniocenteses, citing ethical and religious arguments. She was repeatedly informed about a highly suspected but unidentified syndrome and the related risk of mental retardation. The couple elected to delay for post-natal investigation. The information about this patient was transmitted to the Department of Pediatrics.

The post-natal examination revealed small birth weight ( $2580 \mathrm{~g}$ at 37 weeks), mi- crocephaly (head circumference: $30.5 \mathrm{~cm}$ ), high-arched palate and bilateral broad radially deviated thumbs and abducted hallucis. Neonatal transfontanellar ultrasound and MRI showed a thin and short corpus callosum. Computed tomography scan of the face demonstrated a solitary incisor and a small sinus piriform. Karyotyping found Klinefelter syndrome (47, XXY formulae). The array comparative genomic hybridization testing was normal.

Genetic clinical assessment identified the presence of a prominent columella, short upper lip, low anterior hairline, nevus flammeus at the forehead, simian crease, extranumerary nipple and partially duplicated hallucis. The genetics analysis revealed a transcriptional coactivator CREB-binding protein gene mutation and confirmed the diagnosis of RSTS.

\section{Discussion}

Only three cases of prenatal ultrasound diagnosis or detection have been reported. Two of them have been referred at 21 weeks. Two-dimensional imaging demonstrated the typical findings: broad and abducted thumbs and hallucis and mild micrognathia (Greco E et al. J Ultrasound Med 2009; 28:669-672; Thomas D et al. The foetus.net. 2001). We assessed the inferior facial angle at 17, 22 and 32 weeks, and objectively rejected the presence of micrognathia. Bedeschi et al. report a case detected at 18 weeks, and show 3D images
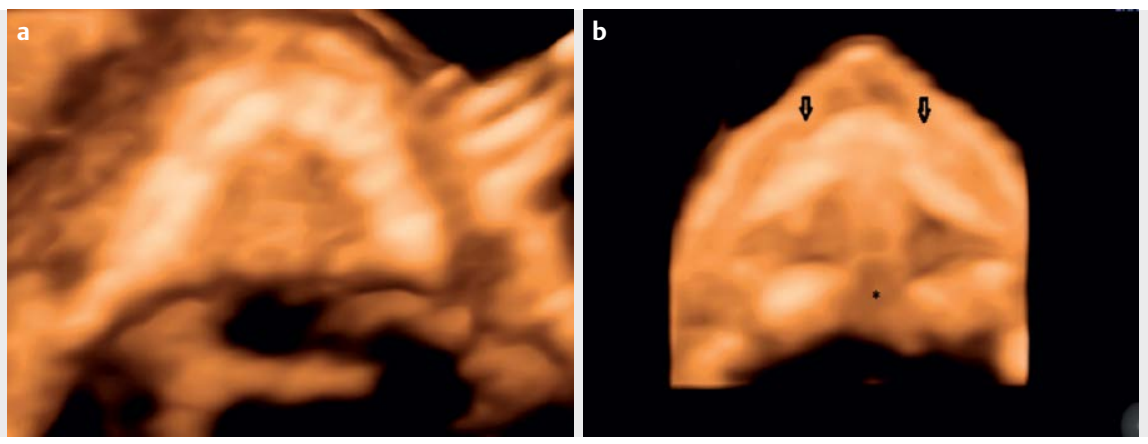

Fig. 1 a) Surface-rendered image of an axial plane of a normal palate at 17 weeks: semi-oval shape. b) High-arched palate at 17 weeks: the palatine process of maxilla and the horizontal palate are misplaced at the level of the intermaxillary suture $\left({ }^{*}\right)$. Note the wide alveolar ridges and the crescent shape $(\downarrow)$. 

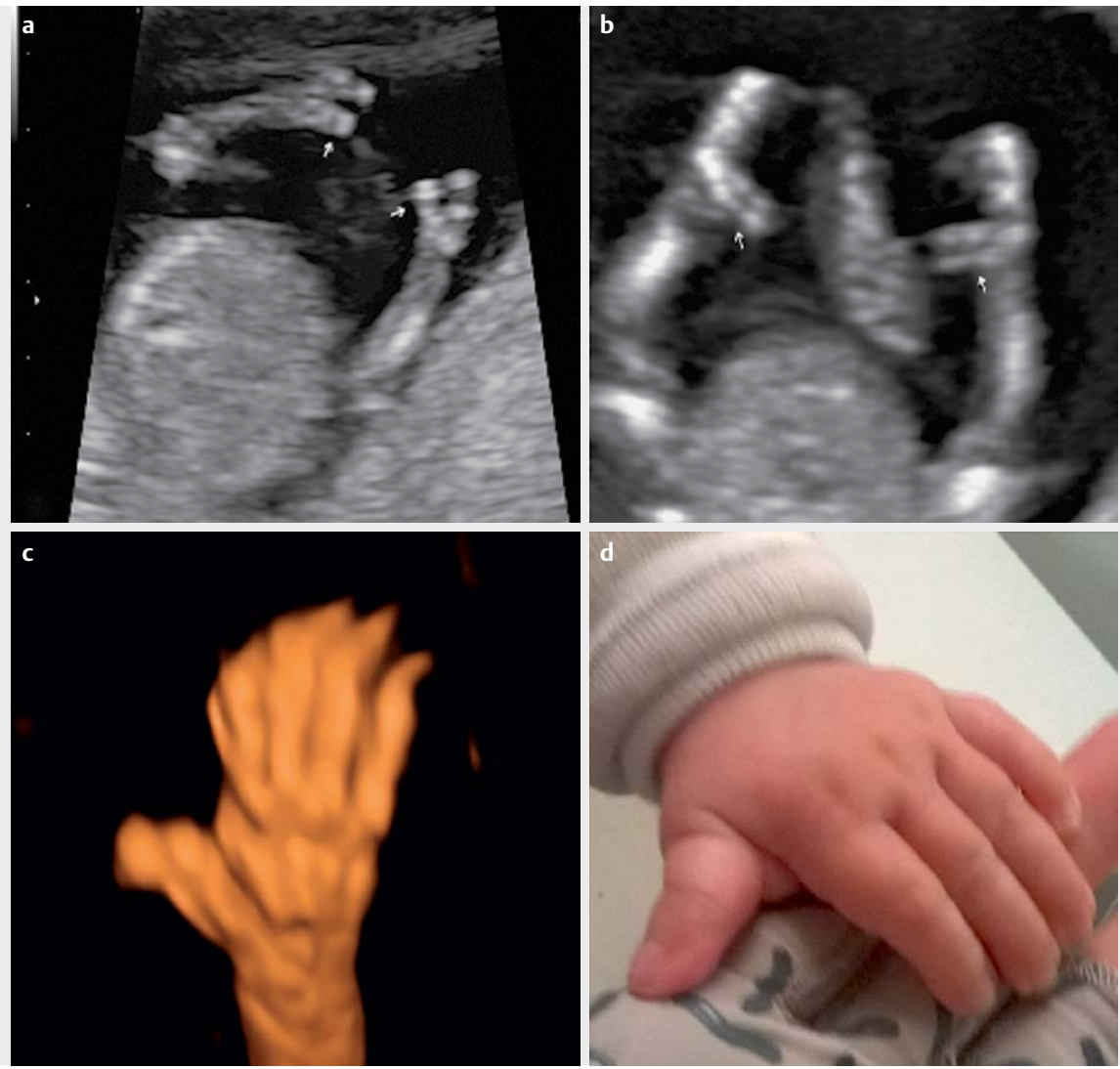

- Fig. 2 Ultrasound image of hands showing abducted and thick thumbs ( $>$ ). a) 2D image at the 12-week scan, showing normal hands b) $2 \mathrm{D}$ image obtained at the 12-week scan, showing unusual thumb angulation. c) Three-dimensional surface-rendered view of the abducted and thick thumb at 17 weeks of gestation (offline analysis). d) Post-natal image of broad radially deviated thumb.

- Table 1 Typical features of Rubinstein-Taybi Syndrome described in this report and in the literature (postnatal series).

\begin{tabular}{|c|c|c|c|c|c|c|}
\hline Anomaly & 12 weeks & 17 weeks & 22 weeks & 32 weeks & Post-natal & Literature \\
\hline Intrauterine growth retardation & - & - & - & + & + & $38 \%{ }^{2}$ \\
\hline Microcephaly & - & - & - & - & + & $94 \% 1$ \\
\hline High-arched palate & - & + & - & - & + & $93 \% 1$ \\
\hline Mild micrognathia & - & - & - & - & - & $75 \% 1$ \\
\hline Protruding nose & - & - & - & - & + & $92 \% 3$ \\
\hline Solitary incisor & - & - & - & - & + & $29 \% 3$ \\
\hline Hypotelorism & - & + & + & + & + & - \\
\hline Broad thumbs/toes & - & + & - & - & + & $100 \% 1$ \\
\hline Partially duplicated halluces & - & - & - & - & + & $16 \% 1$ \\
\hline Radial deviation of the thumbs & + & + & - & - & + & $48 \%^{1}$ \\
\hline Corpus callosum dysgenesis & - & - & + & + & + & $17 \% 1$ \\
\hline Renal malformations & - & - & - & - & - & $52 \%{ }^{2}$ \\
\hline Heart defects & - & - & - & - & - & $33 \% 4$ \\
\hline Cryptorchidism & - & - & - & + & + & $82 \% 1$ \\
\hline
\end{tabular}

1) Rubinstein JH. Am J Med Genet Suppl 1990; 6:3-16; 2) Spena S et al. J Pediatr Genet 2015; 4: 177-186; 3) Bloch-Zupan A et al. Am J Med Genet A. 2007; 143A:570-3; 4) Stevens C et al. Am. J. Med. Genet. 1995; 59: 346-348. 
of the fetal face and thumb at 28 weeks, and MRI of the fetal brain at 25 weeks (Bedeschi et al. Am J Med Genet A 2014; 164:2663-2666). We diagnosed this genetic condition during the post-natal period, but we detected some highly specific and the earliest fetal ultrasound features of the syndrome. As structural anomalies show individual variation and some organ dysfunctions stay undetectable by prenatal ultrasound, hand and face malformations could be uniquely distinctive in numerous genetic disorders ( $\triangleright$ Table $\mathbf{1}$ ).

The postnatal investigations revealed the concomitance of two different genetic syndromes in the same patient: Klinefelter syndrome and RSTS. Although, the positive screening for trisomy 21 could be explained by the XXY-syndrome, the second-trimester anomalous sonographic findings seem to be related to RSTS- a corpus callosum dysgenesis, a high-arched palate and broad thumbs have been reported respectively in $17 \%, 93 \%$ and $100 \%$ of cases (Rubinstein JH. Am J Med Genet Suppl 1990; 6:3-16). The growth restriction and the cryptorchidism are less specific and may also express the presence of Klinefelter syndrome. However, the case series of diagnosed RSTS cited above found an undescended testicle in $82 \%$ of patients and intrauterine growth retardation in $38 \%$.

Based on this report, we demonstrate the advantages of early 3D sonography: the possibility of a retrospective approach in the detection of fetal anomalies, the absence of acoustic shadowing of ossifying fetal structures, and the reconstruction of strictly sagittal views, which offers diagnostic certitude. We are aware that routine fetal morphology is frequently done later in pregnancy and by less experienced sonographers. Nevertheless, this case highlights the diagnostic potential of 3D reconstruction in the early second trimester.

\section{Core Statements}

Subtle hand malformations could be detected even at the first-trimester scan.

Direct ultrasonographic signs of a higharched palate are visible in the early second trimester. We hope that our description of the prenatal appearance of this anomaly will facilitate its identification.

Conflict of Interest
The authors declare that they have no
conflict of interest.
Authors

Iglika Ivancheva Simeonova-Brachot ${ }^{1}$, Laure Gerony-Laffitte ${ }^{2}$
Affiliations

1 Centre Hospitalier de Dax Cote d Argent, Department of Obstetrics and Gynecology, Dax, France

2 Centre Hospitalier de Dax Cote d Argent, Department of Pediatrics, Dax, France

\section{Correspondence}

Dr. Iglika Ivancheva Simeonova-Brachot, MSc Centre Hospitalier de Dax Cote d Argent Department of Obstetrics and Gynecology boulevard Yves du Manoir 40107, Dax

France

Tel.: + 33/627/325 289

iglikasimeonova@yahoo.fr

Bibliography

DOI https://doi.org/10.1055/a-0637-1499 Ultrasound Int Open 2018; 4: E139-E141 (c) Georg Thieme Verlag KG Stuttgart . New York ISSN 2199-7152

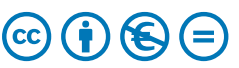

